\title{
Problemas de escritura en la producción de textos de estudiantes de Derecho y Medicina
} Problemas de escrita na produção de textos de estudantes de Direito e Medicina Writing problems in the production of texts of Law
and Medicine students

\author{
Paulina Meza' \\ Melissa Castellón ${ }^{2}$ \\ Jadranka Gladic ${ }^{3}$
}

\section{RESUMEN}

El objetivo es identificar problemas de escritura comunes en textos profesionales con fines académicos producidos por estudiantes de Derecho y Medicina. El corpus estuvo compuesto por 123 textos, que fueron analizados a partir de la aplicación de dos rúbricas diseñadas para evaluar la calidad lingüistico-discursiva. Los resultados evidencian que, en

1. Instituto de Investigación Multidisciplinario en Ciencia y Tecnología. Universidad de La Serena. La Serena - Chile. https://orcid.org/0000-0001-8300-9681. E-mail: pmeza@ userena.cl.

2. Universidad de La Serena. La Serena - Chile. https://orcid.org/0000-0002-1566-3910. E-mail: mcastelloncontreras@gmail.com.

3. Pontificia Universidad Catòlica de Valparaíso. Valparaíso - Chile. https://orcid.org/00000001-6516-5744. E-mail: jadranka.gladic@pucv.cl. 
ambas disciplinas, las dimensiones no logradas corresponden a elementos puramente lingüisticos. Por el contrario, todos los elementos asociados con el conocimiento de la disciplina se encuentran entre las dimensiones logradas. Estos resultados pueden ser útiles para la realización de intervenciones didácticas desde una alfabetización disciplinar.

Palabras-clave: géneros profesionales con fines académicos; alfabetización disciplinar; didáctica de la escritura; problemas de escritura.

\title{
RESUMO
}

O objetivo é identificar problemas comuns de escrita em textos profissionais para fins acadêmicos produzidos por estudantes de Direito e Medicina. O corpus é composto por 123 textos, que foram analisados usando duas rubricas destinadas a avaliar a qualidade linguístico-discursiva. Os resultados mostram que, em ambas as disciplinas, as dimensões não alcançadas correspondem a elementos puramente linguísticos. Pelo contrário, todos os elementos associados ao conhecimento da disciplina estão entre as dimensões alcançadas. Esses resultados podem ser úteis para a realização de intervenções educacionais a partir de uma alfabetização disciplinar.

Palavras-chave: gêneros profissionais para fins acadêmicos; alfabetização disciplinar; didática da escrita; problemas de escrita.

\begin{abstract}
The aim is to identify common writing problems in professional texts for academic purposes produced by students of Law and Medicine. The corpus is composed of 123 texts, which were analyzed using two rubrics designed to assess linguistic-discursive quality. The results show that, the students do not achieve minimum scores in the linguistic elements. In contrast, all participant had more than minimum score in the related to the disciplinary knowledge.These results can be useful for conducting educational interventions from disciplinary literacy.
\end{abstract}

Key-words: professional genres for academic purposes; disciplinary literacy; writing didactics; writing problems. 


\section{Introducción}

La escritura académica hace referencia a un complejo proceso de producción de textos en contextos de formación académica, realizado, en general, por estudiantes u otros miembros de la comunidad, y cuya principal audiencia son los individuos que la componen (Carlino, 2005). Su propósito es construir, deconstruir y reconstruir el conocimiento (Badley, 2009), acciones que implican una serie de habilidades y destrezas muy complejas que no siempre son enseñadas explícitamente durante la formación universitaria.

A lo anterior se suma la dificultad que implica escribir en las disciplinas. Si bien es cierto que las formas de producción de textos en las diversas áreas tienen puntos en común, también es efectivo que poseen características particulares que se ajustan a sus propósitos específicos y a la tradición instaurada en torno a ella (Staples et al., 2016). Estos modos específicos deben ser manejados por cualquier interesado en integrar la comunidad. Al respecto, diversas investigaciones evidencian que la falta de dominio de habilidades de escritura en la educación superior constituye una de las principales causas de reprobación, retraso y deserción de los estudiantes (OlaveArias et al., 2013; Uribe y Carrillo, 2014). Por esta razón, la escritura académica disciplinar debería ser un componente básico en la formación de los estudiantes universitarios, máxime si se considera que, a partir de ella, generalmente, se evalúa y evidencia el manejo de ciertas habilidades disciplinares (Lowe y Zemliansky, 2010).

Con el fin de superar la problemática presentada, varios autores han descrito los errores y dificultades más comunes de los estudiantes de educación terciaria al producir textos académicos (Arechabala et al., 2011; Bassi, 2016; Carlino, 2004; Rodríguez y García 2015; Sabaj, 2009; Sánchez, 2005). Sin embargo, muchas de estas investigaciones han elaborado catastros de dificultades y errores basándose, únicamente, en los resultados de trabajos anteriores o en la experiencia de los investigadores (Carlino, 2004; Rodríguez y García 2015). Asimismo, no han contemplado las diferencias disciplinarias, lo que ha llevado a la generación de listados genéricos de errores en la elaboración de textos académicos (Bassi, 2016; Carlino, 2004; Rodríguez y García 2015; Sabaj, 2009). Además, los escritos considerados en algunos de estos 
estudios han sido analizados desde criterios que se han determinado previamente desde la teoría (Sánchez, 2005) y, solo en algunos casos, desde criterios que emergen desde los mismos textos (Arechabala et al., 2011). Es más, ninguna de las investigaciones mencionadas analizó los textos a partir de instrumentos de evaluación validados y levantados desde las disciplinas.

Considerando la importancia de la disciplinariedad en la actividad escritural en pregrado, nuestro objetivo es identificar los problemas de escritura más comunes en textos profesionales con fines académicos producidos por estudiantes de Derecho y Medicina de dos universidades del Consejo de Rectores de Chile. Para ello, analizamos un corpus constituido por 123 textos. Más concretamente, los problemas de escritura los detectamos a partir de la aplicación de dos rúbricas para analizar la calidad lingüístico-discursiva de los escritos. La elección de las carreras se debe a que los estudiantes ingresantes a ellas requieren los puntajes ponderados más altos de la región y, además, poseen los puntajes de corte más elevados de sus casas de estudio (Departamento de Evaluación, Medición y Registro [DEMRE], 2018). Ello podría incidir en la manera cómo se enfrentan a las tareas de escritura.

Una investigación como la que proponemos se justifica si consideramos que "una forma de acceder a los géneros especializados escritos de la academia es partir desde el supuesto de que los materiales escritos empleados por los estudiantes en su formación universitaria revelan información relevante acerca del discurso y del conocimiento disciplinar" (Parodi, 2007, p. 147). De esta manera, dichos materiales dan cuenta tanto de los tipos de textos que producen como de los rasgos lingüístico-discursivos representativos de su disciplina.

Además, esta investigación se justifica si consideramos que, muchas veces, los estudiantes poseen conocimiento lingüísticodiscursivo general para la elaboración de textos, pero no dominan las prácticas específicas para comunicar el conocimiento en una disciplina particular. En este sentido, este trabajo podría ser útil para sustentar intervenciones didácticas específicas para la producción de textos disciplinares especializados. Ello, considerando que "en la educación superior es absolutamente necesario que los docentes sean capaces de nutrirse de una diversidad de estrategias, técnicas y 
recursos disciplinares para llevar a cabo el proceso de formación de sus estudiantes" (Meza y González-Catalán, 2020, p. 5).

\section{Antecedentes teóricos}

Esta investigación se ubica en un punto intermedio entre el discurso académico y el profesional, puesto que los textos que analizamos son de tipo profesional, pero se producen en una esfera académica. Por una parte, el discurso profesional es aquel que se genera al interior de un campo profesional determinado entre los integrantes de una misma profesión, de profesiones distintas o entre los participantes de una comunidad profesional y un público lego (Gunnarsson, 2004; Parodi, 2007). Entendemos como discurso profesional, entonces, toda forma de comunicación que circula en una actividad profesional concreta que responde a las características lingüístico-discursivas específicas requeridas por dicha actividad.

Por otra parte, el discurso académico tiene como propósito cristalizar y difundir un conocimiento disciplinar y, también, apoyar la preparación de los miembros en formación de una comunidad de especialistas (Parodi, 2007). Se caracterizaría por rasgos como la precisión, la claridad y la objetividad (Montolío, 2000; Parodi, 2007, entre otros). Así, el concepto en cuestión remite al conjunto de producciones, ya sea en forma oral o escrita, realizadas en contexto de enseñanza-aprendizaje, por lo que involucra a investigadores, docentes y estudiantes (Hall y López, 2011). La definición de discurso académico, por tanto, se acota a los textos utilizados durante el proceso de formación de enseñanza superior.

Considerando el tipo de textos que aquí analizamos y el hecho de que se ha reconocido una frontera difusa entre los géneros académicos y profesionales (Parodi, 2009), proponemos el concepto de géneros profesionales con fines académicos. En nuestra concepción, estos géneros son aquellos que circulan en esferas académicas universitarias, pero cuyas características son propias de géneros vinculados al ámbito profesional. El fin de los textos asociados a estos géneros es, generalmente, de índole didáctica, pues se utilizan como instrumento de formación de los estudiantes de una carrera específica para prepararlos 
para la vida profesional, de modo que constituyen una herramienta para acompañar el transitar adecuado desde el mundo académico hacia el laboral.

Dado que no encontramos en la bibliografía consultada el concepto propuesto, para su caracterización consideramos los criterios y variables clasificatorios de los géneros planteados por Parodi et al. (2010). Sobre esta base, las características de los géneros profesionales con fines académicos son:

Tabla 1 - Caracterización géneros profesionales con fines académicos

\begin{tabular}{ll}
\hline Criterio & Géneros profesionales con fines académicos \\
\hline Macropropósito comunicativo & Instruir \\
\hline Relación entre los participantes & Escritor semilego, lector experto \\
\hline Contexto ideal de circulación & Pedagógico-disciplinar (Educación superior) \\
\hline Modo de organización del discurso & Argumentativo, narrativo, descriptivo \\
\hline Modalidad & Multimodal \\
\hline
\end{tabular}

Finalmente, el último concepto que delimitamos es el de calidad lingüístico-discursiva. Si bien no existe en la bibliografía revisada una definición para este constructo, diversos investigadores (Errázuriz, 2014; Rico y Níkleva, 2016; Tapia et al., 2003, entre otros) concuerdan al considerar la presencia de un conjunto de características para dar cuenta de la calidad discursiva de un texto. Así, por ejemplo, en términos generales, se han considerado elementos en el plano oracional, estructural y normativo (Arechabala et al., 2011; Bustos, 2011; Gaibani, 2015; Tapia et al., 2003). En esta investigación, entendemos la calidad lingüístico-discursiva como los atributos textuales y discursivos que, según los miembros expertos de una comunidad específica, debe poseer un texto de tipo profesional con fines académicos de una disciplina concreta. Dichos atributos permitirán juzgar su valor en relación con criterios preestablecidos. Algunos de ellos son, por ejemplo, el léxico especializado, la construcción de oraciones, la adecuación a la audiencia, entre otros. 


\section{Marco metodológico}

El objetivo de esta investigación es identificar los problemas de escritura más frecuentes en textos profesionales con fines académicos producidos por estudiantes de Derecho y Medicina. Este se concreta a partir de una investigación de corte cualitativo y alcance descriptivo (Hernández et al., 2006).

El corpus de investigación, denominado TeProFAc (Textos Profesionales con Fines Académicos), está constituido por dos subcorpus, uno del área del Derecho (TeProFAc-D) y otro del área de la Medicina (TeProFAc-M). El subcorpus de Derecho está constituido por 65 textos pertenecientes a distintos géneros (Testamento, Contestación de demanda, entre otros), que suman 540.246 palabras. Mientras que el de Medicina está compuesto por 58 textos (Ficha Clínica y Estudio Familiar), cuyo número de palabras asciende a 242.242. En definitiva, TeProFAc se constituye de 123 textos, que suman 782.488 palabras. Es importante señalar que todos los textos fueron producidos por estudiantes de Derecho y Medicina en distintos cursos de su proceso de formación de pregrado.

En cuanto a los procedimientos de análisis, ellos se sintetizan en la siguiente figura:

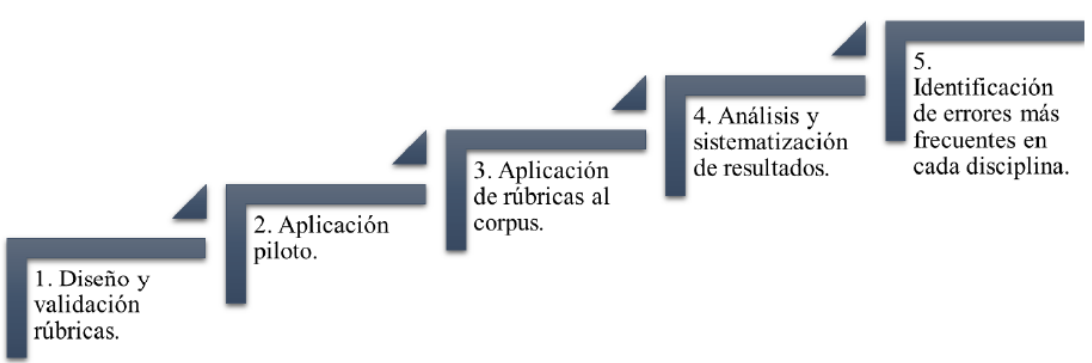

Figura 1 - Síntesis de procedimientos de análisis

Como se observa en la Figura 1, el primer procedimiento fue el diseño y validación de las rúbricas para evaluar la calidad lingüísticodiscursiva de los textos del corpus. Dada la intención de construir este instrumento sobre la base de los requerimientos de cada disciplina, su diseño requirió algunos pasos previos: a) revisión de insumos de cada 
área (textos producidos por los estudiantes, instrumentos de evaluación utilizados por los docentes); b) aplicación de cuestionarios a los profesores; y c) entrevistas en profundidad a los docentes.

Tras ello, un equipo interdisciplinario construyó un instrumento de evaluación para cada disciplina: RECLiTeD (Rúbrica para evaluar la calidad lingüístico-discursiva en Derecho), presentada en Meza y González (2021); y RECLiTeM para Medicina (Meza y GonzálezCatalán, 2020). Ambos instrumentos, por separado, fueron validados exitosamente en distintas etapas: a) revisión por parte de expertos en escritura académica y en evaluación; b) validación de contenido por jueces expertos de distintas nacionalidades y con diferentes perfiles profesionales; y c) aplicación piloto. Para calcular el resultado de b y c, se utilizaron dos índices: porcentaje de acuerdo y coeficiente Kappa de Fleiss (1971). Los resultados numéricos finales de esta etapa de validación son los siguientes:

Tabla 2 - Resultados juicio de pares y aplicación piloto de las rúbricas

\begin{tabular}{llll}
\hline & & RECLiTeD & RECLiTeM \\
\hline \multirow{2}{*}{ Juicio de pares } & Porcentaje de acuerdo & $72,5 \%$ & $63,16 \%$ \\
\cline { 2 - 4 } & Coeficiente Kappa de Fleiss & 0,7 & 0,6 \\
\hline \multirow{2}{*}{ Aplicación piloto } & Porcentaje de acuerdo & $61,67 \%$ & $90,2 \%$ \\
\cline { 2 - 4 } & Coeficiente Kappa de Fleiss & 0,6 & 0,9 \\
\hline
\end{tabular}

Como se ve en la Tabla 2, los resultados permitieron validar exitosamente nuestros instrumentos, pues, de acuerdo con la interpretación del coeficiente Kappa de Fleiss (1971) ofrecida por Landis y Koch (1977), la rúbrica de Derecho obtuvo un acuerdo considerable en el juicio de pares y en el pilotaje. Mientras que el instrumento de Medicina resultó con un acuerdo considerable en el juicio de pares y con un acuerdo casi perfecto en la aplicación piloto. Para mayor detalle, sugerimos revisar los artículos en los que se presentan ambas rúbricas (Meza y González-Catalán, 2020; Meza y González, 2021). En estos artículos, se definen todas las dimensiones y macrodimensiones que constituyen los instrumentos; se describen detalladamente los procedimientos para la construcción y validación de las rúbricas; y, además, se sustenta teóricamente su construcción. 
Cabe agregar que el pilotaje realizado sirvió como método de calibración entre evaluadoras, de forma que la evaluación de los textos con las rúbricas fuese lo más similar posible. Para el caso de Derecho, participaron dos anotadoras que analizaron, a modo de prueba, seis textos; mientras que en Medicina contamos con tres evaluadoras, quienes aplicaron la rúbrica a tres textos. En ambos casos, las anotadoras aplicaron el instrumento de forma individual para, luego, cotejar los resultados grupalmente, evaluar las diferencias y despejar dudas. De acuerdo con los resultados presentados en la Tabla 2, este pilotaje fue exitoso.

El siguiente procedimiento fue la aplicación de los instrumentos validados para cada subcorpus. En Derecho, fue realizada por dos anotadoras y en Medicina participaron tres. En ambos casos, contamos con el apoyo de expertos disciplinares para asistirnos en dudas específicas. Las rúbricas diseñadas y aplicadas se sintetizan en la Tabla 3:

Tabla 3 - Síntesis dimensiones y macrodimensiones RECLiTeD y RECLiTeM

\begin{tabular}{|c|c|c|}
\hline & RECLiTeD & RECLiTeM \\
\hline \multirow{3}{*}{$\begin{array}{l}\text { I. Situación } \\
\text { Comunicativa }\end{array}$} & 1. Audiencia & 1. Destinatario \\
\hline & 2. Tema & 2. Tema \\
\hline & 3. Propósito & 3. Propósito \\
\hline \multirow{6}{*}{$\begin{array}{l}\text { II. Comunicación } \\
\text { del conocimiento } \\
\text { jurídico/ } \\
\text { Comunicación } \\
\text { del conocimiento } \\
\text { especializado en } \\
\text { Medicina }\end{array}$} & 4. Léxico jurídico & 4. Léxico médico \\
\hline & $\begin{array}{l}\text { 5. Organización de la } \\
\text { información }\end{array}$ & $\begin{array}{l}\text { 5. Estructura interna: modalidad } \\
\text { expositiva }\end{array}$ \\
\hline & $\begin{array}{l}\text { 6. Sustento o exposición } \\
\text { jurídica }\end{array}$ & $\begin{array}{l}\text { 6. Estructura interna: modalidad } \\
\text { argumentativa }\end{array}$ \\
\hline & 7. Marcas de posicionamiento & $\begin{array}{l}\text { 7. Tratamiento de la información en } \\
\text { modalidad expositiva }\end{array}$ \\
\hline & & $\begin{array}{l}\text { 8. Tratamiento de la información en } \\
\text { modalidad argumentativa }\end{array}$ \\
\hline & & $\begin{array}{l}\text { 9. Relación entre los distintos sistemas } \\
\text { semióticos }\end{array}$ \\
\hline \multirow{6}{*}{$\begin{array}{l}\text { III. Estructura } \\
\text { global y oracional }\end{array}$} & 8. Construcción de oraciones & 10. Organización textual en párrafos \\
\hline & $\begin{array}{l}\text { 9. Organización textual en } \\
\text { párrafos }\end{array}$ & 11. Cohesión interoracional \\
\hline & $\begin{array}{l}\text { 10. Estructura interna de los } \\
\text { párrafos }\end{array}$ & 12. Coherencia interpárrafo \\
\hline & 11. Cohesión intrapárrafo & 13. Construcción de frases y oraciones \\
\hline & 12. Coherencia interpárrafo & 14. Secuencias textuales \\
\hline & 13. Secuencias textuales & \\
\hline \multirow[t]{3}{*}{ IV. Normativa } & 14. Normas gramaticales & 15. Puntuación \\
\hline & 15. Puntuación & 16. Normas sintácticas \\
\hline & $\begin{array}{l}\text { 16. Normas de ortografía } \\
\text { literal y acentual }\end{array}$ & $\begin{array}{l}\text { 17. Normas de ortografía literal y } \\
\text { acentual }\end{array}$ \\
\hline
\end{tabular}


Cada rúbrica cuenta con diferentes niveles de desempeño que permiten evaluar los textos del corpus. En las Figuras 2 y 3 , se presentan ejemplos de ello:

\begin{tabular}{|c|c|c|c|c|}
\hline \multirow[t]{2}{*}{ 4. Léxico jurídico } & 0 punto & 2 puntos & 4 puntos & \multirow[b]{2}{*}{$\begin{array}{l}6 \text { puntos } \\
\text { Integra de manera adecuada, en todo } \\
\text { el texto, terminologia técnica de la } \\
\text { disciplina, incorporando los conceptos } \\
\text { especializados en construcciones que } \\
\text { permiten comunicar su significado. }\end{array}$} \\
\hline & $\begin{array}{l}\text { Integra de manera adecuada la } \\
\text { terminología técnica de la disciplina, } \\
\text { incorporando los conceptos } \\
\text { especializados en construcciones que } \\
\text { permiten comunicar su significado, } \\
\text { sin embargo, presenta cinco o más } \\
\text { errores en el texto. }\end{array}$ & $\begin{array}{l}\text { Integra de manera adecuada la } \\
\text { terminología técnica de la disciplina, } \\
\text { incorporando los conceptos } \\
\text { especializados en construcciones que } \\
\text { permiten comunicar su significado, sin } \\
\text { embargo, presenta tres o cuatro } \\
\text { erroresen el texto. }\end{array}$ & $\begin{array}{l}\text { Integra de manera adecuada la } \\
\text { terminologia técnica de la disciplina, } \\
\text { incorporando los conceptos } \\
\text { especializados en construcciones que } \\
\text { permiten comunicar su significado, sin } \\
\text { embargo, presenta uno o dos errores en } \\
\text { el texto. }\end{array}$ & \\
\hline
\end{tabular}

Figura 2 - Niveles de desempeño para la dimensión "Léxico jurídico" (RECLiTeD)

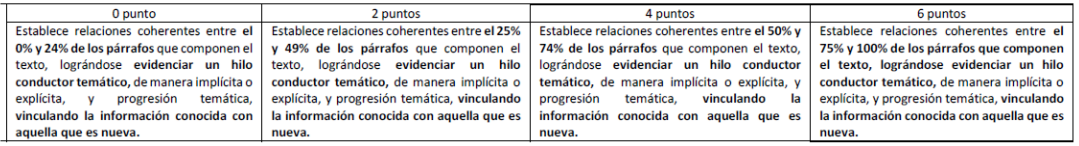

Figura 3 - Niveles de desempeño para la dimensión "Coherencia interpárrafo" (RECLiTeM)

Una vez aplicadas las rúbricas, se realizó el análisis y sistematización de los resultados. Para ello, fijamos un umbral de $60 \%$ como nivel de logro para diferenciar las dimensiones logradas de las no logradas. Con ello, detectamos los problemas de escritura, que son los resultados que presentamos en el apartado siguiente. El establecimiento de dicho umbral obedece a dos razones, primero, porque en las carreras incluidas en la investigación el nivel de exigencia de aprobación para los estudiantes es de $60 \%$. Y, segundo, porque dicho umbral ha sido escogido en otras investigaciones en las que se estudian distintos géneros académicos (Artículo de Investigación, Tesis y Texto Disciplinar), para establecer el estatus de una categoría (Ibáñez, 2008; Kanoksilapatham, 2007; Martínez 2015; Meza y da Cunha, 2019).

\section{Análisis y discusión de resultados}

En primer lugar, tras la aplicación de RECLiTeD, los resultados obtenidos para Derecho son los que se presentan, a continuación, en el Gráfico 1: 


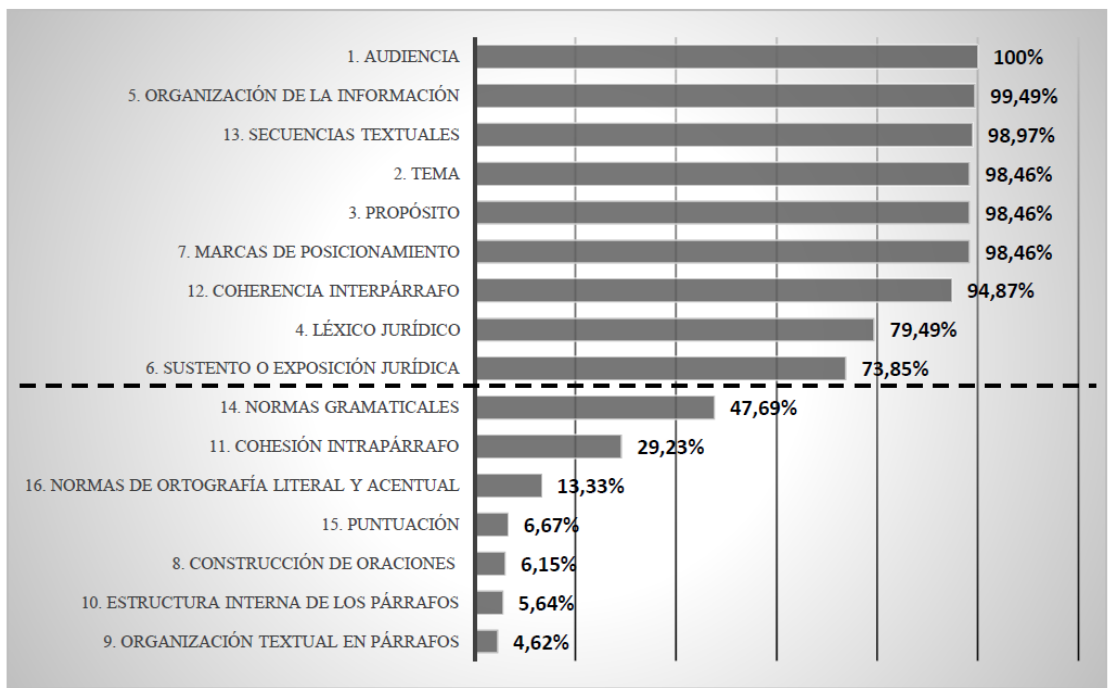

Gráfico 1 - Promedio de porcentajes de logro de la calidad lingüístico-discursiva general en Derecho

La línea punteada del gráfico separa las dimensiones logradas (sobre el 60\%) de aquellas no logradas. Como se puede observar, los textos de Derecho alcanzan este porcentaje de logro en 9 de las 16 dimensiones contempladas en la rúbrica $(56,25 \%)$. En consecuencia, el umbral establecido es superado ampliamente en la gran mayoría de las dimensiones establecidas, de hecho, se supera el 90\% de logro en 7 de ellas; e, incluso, en el ítem "Audiencia", todos los textos obtienen el puntaje máximo ( $100 \%$ de logro), situación que revela que todos los textos son adecuados a las características o requerimientos de su destinatario. Todo ello, por supuesto, de acuerdo con los aspectos y dimensiones esperadas en la disciplina en cuestión.

Los estudiantes evidencian excelente dominio también en los otros dos componentes de la situación retórica: "Tema" y "Propósito" (98,46\% de logro). Un caso similar es el del ítem que dice relación con la organización de la información de acuerdo con los requerimientos del género producido $(99,49 \%)$ y de aquel que hace referencia al uso adecuado de secuencias textuales en el desarrollo del texto $(98,97 \%)$. Se aprecia un muy buen desempeño también en las marcas de posicionamiento incorporadas $(98,46 \%)$. Por último, la dimensión 
"Coherencia interpárrafo" también supera el 90\% de logro, lo que implica que los textos poseen párrafos bien conectados. Este ítem es interesante, pues es uno de los pocos elementos de la dimensión "Estructura global y oracional" en el que se advierte un desempeño sobresaliente.

En esta línea, llama la atención la disparidad existente entre algunas dimensiones que parecen muy cercanas teóricamente, pero que, en la práctica, evidencian desempeños muy diferentes en los textos analizados. Esto ocurre, por ejemplo, entre la dimensión "Coherencia Interpárrafo", que marca un $94,87 \%$ de logro, y las dimensiones "Estructura interna de los párrafos", que apenas alcanza un 5,64\% de logro, y "Cohesión intrapárrafo", que logra un 29,23\%. Ello indica que los estudiantes de Derecho, en la mayoría de los casos, son capaces de establecer relaciones coherentes entre los párrafos que componen sus textos, de evidenciar un hilo conductor temático, de manera implícita o explícita, y de desarrollar una progresión temática adecuada. Sin embargo, muy pocas veces son capaces de construir párrafos adecuados o de utilizar mecanismos de cohesión apropiados dentro de estos. Esto podría deberse a que las habilidades que se requieren para construir párrafos adecuados requieren de un conocimiento lingüístico más especializado. Otra explicación complementaria radica en el hecho de que, en los textos de Derecho, identificamos con bastante frecuencia párrafos muy extensos. Este hecho podría derivar, por ejemplo, en que se pierda el referente o se generen repeticiones innecesarias.

Por otro lado, aunque no de forma tan determinante como en los casos comentados, "Léxico jurídico" y "Sustento o exposición jurídica" son dimensiones en las que los textos superan el porcentaje mínimo de logro. Ello evidencia, por una parte, que en los textos se integra de manera adecuada la terminología técnica de la disciplina; y, por otra, que se realiza un buen trabajo en la exposición del contenido o en la fundamentación de la tesis u opinión jurídica, según corresponda.

Dada la pretensión didáctica de este trabajo, nos focalizaremos en aquellas dimensiones que no alcanzaron el porcentaje mínimo de logro, de modo que los problemas identificados puedan servir de insumo para una didáctica de la escritura en las disciplinas estudiadas. Para ello, además, ejemplificaremos cada situación con casos reales del corpus. 
Identificamos estos ejemplos con el código del texto al que pertenecen, destacamos con cursiva los fragmentos relevantes y ocultamos en ellos cualquier tipo de información sensible.

En esta línea, los dos principales problemas al producir textos propios del desempeño profesional de un abogado dicen relación con la construcción de párrafos, concretamente, con las dimensiones "Organización textual en párrafos" (4,62\% de logro) y "Estructura interna de los párrafos" (5,64\%). Ello implica que existe gran dificultad para elaborar un texto en el que la mayoría de sus párrafos cuenten, por una parte, con, al menos, dos oraciones claramente identificables y cuya extensión permita un desarrollo claro y completo de las ideas integradas; y, por otra, con una oración temática claramente identificable junto con una serie de oraciones de apoyo coherentes que nutren la idea principal. Ejemplos de este problema son los párrafos:

[1] Por tanto, ruego a U.S. tenerlo presente [DO_UCN_37].

[2] "En este contexto, durante las visita realizada al Complejo Penitenciario de La Serena con fecha 8 de octubre de 2018, enmarcada en el ejercicio de las atribuciones contenidas en la Ley $\mathrm{N}^{\circ} 20.405$, que faculta a los funcionarios y funcionarias del INDH para ingresar a recintos donde se encuentren personas privadas de libertad, funcionarios de la sede regional de Coquimbo del INDH han tomado conocimiento de un número importante personas privadas de libertad, todas del módulo 57 de dicho establecimiento penitenciario, que acusan dormir conjuntamente dos o más de ellas en un mismo colchón, lo cual daría cuenta de una situación de hacinamiento al interior de dichas celdas"[DO_UCN_40].

Como se puede ver en DO_UCN_37 y DO_UCN_40, el problema central es la presentación de párrafos unioracionales, cuyas oraciones pueden ser muy breves [1] o muy extensas [2]. Este segundo caso nos parece más preocupante, pues podría dificultar la comprensión por parte del lector o llevarlo a conclusiones erradas sobre el contenido del texto. Según Muñoz (2017), el origen del párrafo unioracional, en la redacción jurídica tradicional, se impuso como norma en las sentencias; sin embargo, como vemos en los ejemplos, este tipo de párrafos ha traspasado las fronteras de dicho género. Ello derivó en párrafos inmensos e incomprensibles, que, muchas veces, ni los profesionales del Derecho podían entender (Muñoz, 2017). En esta línea, los profesores 
de Derecho Gidi y Weihofen (2018), si bien reconocen que no existe una extensión ideal para los párrafos en el ámbito jurídico, sugieren párrafos breves, constituidos por, al menos, dos oraciones y no más de diez líneas. Esto, ya que los párrafos breves contribuyen a que el escritor produzca ideas de formas clara y precisa; y, también, a que el lector realice un menor esfuerzo para comprender.

El tercer lugar entre los errores más frecuentes lo ocupa el ítem "Construcción de oraciones" (6,15\% de logro promedio). Este evalúa que la mayoría de las oraciones que construye el estudiante estén constituidas por un referente claramente identificable y dos o tres ideas que lo nutran. Un ejemplo de este problema es:

[3] Que con fecha 3 de abril del 2018 en la segunda sala del juzgado de letras del trabajo de la ciudad de la Serena, dándole curso a la audiencia preparatoria de juicio y una vez realizada la actuación por parte del tribunal a la que se refiere el numeral primero del artículo 453 del código del trabajo, es decir, la relación breve y somera que debe hacer el juez de letras del contenido de la demanda y la contestación, antes del llamado a conciliación por parte del órgano jurisdiccional, el juzgador doña XXX, señala lo siguiente "llamo a la demandante a desistirse o a llegar a un acuerdo con la otra parte, pues yo ante este tipo de demandas en el noventa por ciento fallo a favor del demandado", ante este acontecimiento [...] veredicto alguno sobre lo que se discute [DO_UCN_61]

Por razones de espacio hemos recortado el ejemplo, que, originalmente, corresponde a una sola oración constituida por 17 líneas, con múltiples problemas de puntuación. Tal como en el caso de los párrafos, este tipo de oraciones dificulta la comprensión del lector y, también, genera que el escritor pierda la claridad y precisión en la redacción del texto que, en este caso, corresponde a un Recurso de nulidad. Por otro lado, el hecho de presentar oraciones tan extensas deriva en que, en ocasiones, haya una pérdida del referente, hecho que dificulta aún más la comprensión por parte del lector $\mathrm{y}$, además, puede dar origen a malentendidos.

En la cuarta posición de los problemas más frecuentes, se encuentran aquellos asociados a la puntuación (6,67\% de logro). En esta dimensión, se evaluó el empleo adecuado de los signos de puntuación, de acuerdo con las normas de la lengua española. Por ejemplo: 
[4] "A estos hechos debe agregársele la circunstancia que la compradora, es sobrina de don XXX, por lo que no podría desconocer la calidad de cónyuges entre mi representada y el fallecido cónyuge, dicho de otra forma, los hechos permiten tener por cierto el conocimiento de la calidad de cónyuges de la compradora, respecto a quiénes serían sus tíos" [DO_UCN_42].

En el ejemplo, aparecen dos problemas muy frecuentes en el corpus de Derecho, a saber, el uso de coma entre sujeto y verbo y la utilización de coma donde corresponde un punto seguido. Esto es preocupante, pues, de acuerdo con Alcaraz et al. (2014), los errores más habituales en los escritos forenses se deben al mal uso de los signos de puntuación, especialmente, la coma. Este último, según los mismos autores, es causante de algunos problemas de interpretación.

Luego, en la quinta posición, con un 13,33\% de logro, aparecen los problemas asociados a las normas de ortografía literal y acentual. Un ejemplo es:

[5] "el producto de nuestra representada seria perteneciente a la clase 30 o servicios de la clase 44 (servicios de apicultura)" [DO_UCN_35].

Con respecto a este punto, es importante señalar que, en el corpus de Derecho, predominan ampliamente los errores asociados a la ortografía acentual más que a la literal. La baja frecuencia de errores de este último tipo podría deberse a que, en general, estos estudiantes poseen un bagaje lingüístico superior a los de otras áreas, lo que se evidencia, por ejemplo, en su puntaje de ingreso en la Prueba de Selección Universitaria (DEMRE, 2018). Para el caso de la ortografía acentual, según explica Valencia (2014), el uso de la tilde sugiere una serie de operaciones cognitivas para ser usado correctamente. Sin embargo, muchas veces, dentro de las mallas curriculares de Derecho no hay un espacio dedicado a desarrollar estas habilidades, pues se da por sentado que son conocimientos que debiesen traer consigo los estudiantes o porque, dentro de dicha carrera, aspectos como la ortografía, son considerados superficiales (Araujo, 2018). Esto explicaría los resultados obtenidos y la poca preparación con la que cuentan los estudiantes en este aspecto al momento de producir un texto. 
Otra dimensión en la que los textos evaluados no alcanzan el 60\% de logro establecido es "Cohesión intrapárrafo". Ella apunta a evaluar el buen uso de mecanismos de cohesión (pronominalización, correferencia, deixis, marcadores discursivos, etc.) para establecer relaciones entre los segmentos que componen los párrafos. Un ejemplo es:

[6] "puesto que esta expresión tiene un origen francés que, en español, significa natural, expresión utilizada por nuestra representante debido al origen franco-chileno que tiene la empresa de nuestra representada" [DO_UCN_35].

En el ejemplo, se identifica la repetición de palabras iguales o muy similares, lo que da cuenta de un problema en el uso de la correferencia, puesto que, en menos de 3 líneas, el estudiante repite dos veces la palabra "expresión" y otras dos veces lo hace con palabras muy similares (representante y representada). Otra situación frecuente detectada en esta dimensión es la repetición constante de la conjunción "y", lo que podría sugerir desconocimiento de una variedad más amplia de marcadores discursivos. Ambos errores dicen relación con el uso de mecanismos de cohesión. Es, precisamente, debido a los problemas que se han detectado en el uso de dispositivos de cohesión por parte de los estudiantes que se ha recomendado su enseñanza explícita para, así, ayudarlos a alcanzar una competencia discursiva acorde con las expectativas de cada comunidad (Ahmad, 2019).

La dimensión "Normas gramaticales" es la última en que los textos de Derecho no alcanzan el 60\% de logro. Ella apunta a la elaboración de un texto gramaticalmente correcto, vale decir, que no incurre en vicios idiomáticos, tales como: discordancia, queísmo, dequeísmo, hipérbaton, barbarismo, cacofonía, etc. Ejemplo de este tipo de problema es:

[7] "Que sólo se reconocen como hechos no controvertidos la circunstancia de que la trabajadora se encontraba vinculada bajo una relación de subordinación y dependencia en calidad de secretaria entre el 1 de marzo de 2014 y el 6 de agosto de 2018" [DO_UCN_53].

Como se evidencia en el ejemplo, algunos de los problemas más recurrentes en el ámbito comentado es la concordancia y, también, el queísmo y dequeísmo. La explicación de la presencia de este tipo de errores se relaciona con lo mencionado anteriormente respecto 
al desconocimiento y el poco trato de las normas gramaticales en la enseñanza superior. Así, en el nivel académico universitario, se asumen como dominados todos los procedimientos, técnicas y estrategias vinculados a la escritura y trabajados a lo largo de la vida escolar (Rico y Nikleva, 2016). Sin embargo, en nuestra experiencia, este tipo de fenómenos lingüísticos no son estudiados con detenimiento en los años de escolaridad en Chile.

En síntesis, a partir de la información presentada en el Gráfico 1, podemos afirmar que los tres problemas más frecuentes identificados en Derecho se pueden agrupar bajo la macrodimensión "Estructura global y oracional", así como también el sexto tipo error más habitual (“Cohesión intrapárrafo"), que, igualmente, se ubica en esta área. Por su parte, los errores que ocupan la cuarta, quinta y séptima posición de mayor reiteración se agrupan en la macrodimensión "Normativa". En cuanto a las dimensiones logradas, todas ellas pertenecen a las macrodimensiones "Situación comunicativa" y "Comunicación del conocimiento jurídico", que no presentan ninguna dimensión no lograda. Además, las únicas dimensiones logradas del grupo "Estructura global y oracional" son "Coherencia interpárrafo" y "Secuencias textuales".

A continuación, en el Gráfico 2, se presentan los resultados obtenidos del análisis de los textos de Medicina:

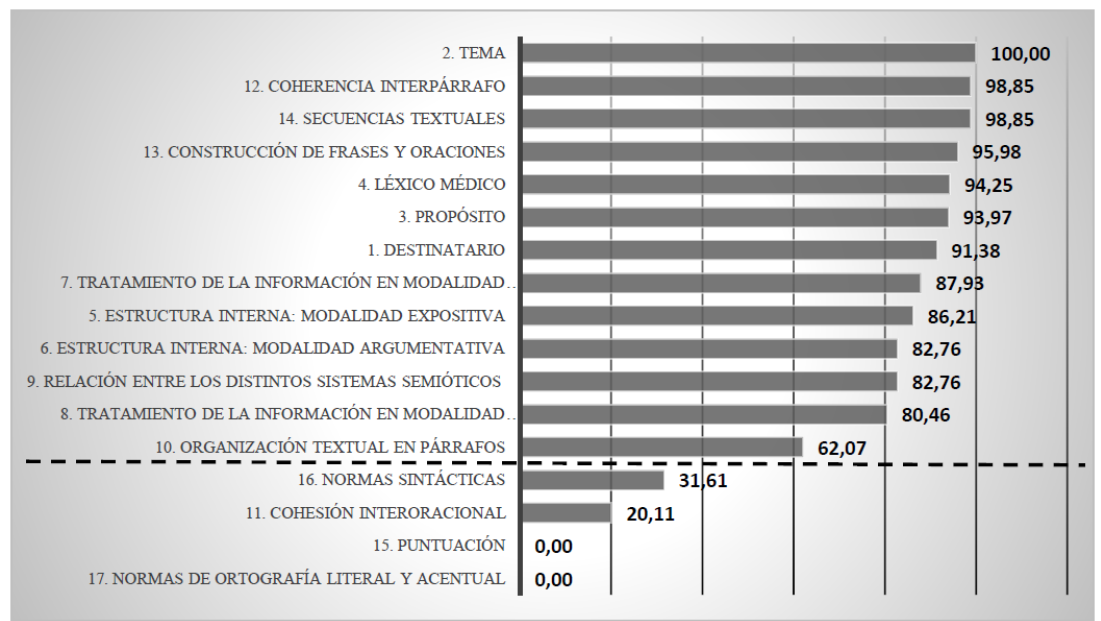

Gráfico 2 - Promedio de porcentajes de logro de la calidad lingüístico-discursiva general en Medicina 
En el caso de Medicina, como muestra el Gráfico 2, 13 de las 17 dimensiones $(76,47 \%)$ alcanzan un porcentaje de logro que supera el 60\%. Como ocurría en Derecho, todos los ítems asociados a la situación retórica se ubican en este grupo, de hecho, la dimensión "Tema" alcanza el 100\% de logro en todos los textos de Medicina. Se advierte un excelente desempeño también en todas las dimensiones que dicen relación con la comunicación del conocimiento especializado en Medicina. Ellas son, primero, "Léxico médico" (94,25\%), que corresponde al uso de conceptos especializados de Medicina. Segundo, los ítems que se asocian con la estructuración y presentación de la información en las modalidades expositiva y argumentativa, concretamente: "Estructura interna: modalidad expositiva" (86,21\%), "Estructura interna: modalidad argumentativa" (82,76\%), "Tratamiento de la información en modalidad expositiva" $(87,93 \%)$ y "Tratamiento de la información en modalidad argumentativa" (80,46\%). Y, tercero, la dimensión que evalúa la relación entre los distintos sistemas semióticos, esto es, que ellos se relacionen de forma lógica y coherente, de acuerdo con los requerimientos del género y su propósito $(82,76 \%)$.

Luego, en la macrodimensión que tiene que ver con la estructura global y oracional, 4 de los 5 ítems se encuentran sobre el 60\% de logro. Ellos son: "Organización textual en párrafos" $(62,07 \%)$, "Coherencia interpárrafo" (98,85\%), “Construcción de frases y oraciones” (95,98\%) y "Secuencias textuales" (98,85\%). Los puntajes obtenidos en esta macrodimensión demuestran que los estudiantes son capaces de construir párrafos, oraciones y frases que son comprensibles para el lector, ya que cuentan con referentes que son identificables y poseen una extensión que permite al lector reconocer ideas claras y precisas. Sin embargo, y como se verá más adelante, dentro de esta macrodimensión, al igual que en el área del Derecho, los estudiantes no alcanzan un puntaje satisfactorio en el ítem "Cohesión interoracional", es decir, no utilizan adecuadamente mecanismos de cohesión. El uso de este tipo de mecanismos debiese enseñarse explícitamente a los estudiantes, con el propósito de que sus textos sean adecuados a los requerimientos de su comunidad disciplinar (Ahmad, 2019).

En cuanto a las dimensiones no logradas, advertimos que ellas son cuatro, tres de las cuales pertenecen a la macrodimensión "Normativa" y una a "Estructura global y oracional". Ello implica que todos los ítems 
asociados a la normativa de la lengua española están bajo el nivel de logro establecido. Esto es aún más preocupante si observamos que las dimensiones "Puntuación" y "Normas de ortografía literal y acentual", que son las dos más problemáticas, tienen un porcentaje igual a cero, lo que evidencia que todos los textos analizados obtuvieron el puntaje más bajo en dichos aspectos. Ejemplos de errores de ortografía [8] y puntuación [9] son:

[8] Cejas conservadas, simétricas, sin signo de la cola. Parpados de motilidad conservada, apertura y cierre completos, sin lesiones [MED_UCN_30].

[9] En primer lugar se observa que todas las figuras que representan a los integrantes de su familia fueron dibujadas dentro del círculo [MED_ UCN_20].

Son varias las causas que podrían explicar este tipo de problemas. Primero, la presencia de errores ortográficos y de puntuación es un fenómeno transdisciplinar (Cofré, 2019; Melguizo, 2016), que, además, no es exclusivo de la Educación Superior (Sotomayor et al., 2013). Segundo, existen otras concepciones que atribuyen este fenómeno a la existencia de un número considerable de profesores especialistas en ciencias, quienes carecen de las herramientas necesarias para enseñar ortografía y redacción, pese a su autopreparación y buen empeño por hacerlo eficazmente (Pérez et al., 2012). Tercero, como señala explícitamente uno de los docentes de Medicina entrevistados para esta investigación, estos aspectos son irrelevantes en la formación de los médicos, por lo que no se les da ninguna importancia, ya que se asume que serán malos escritores (Meza y González-Catalán, 2020). Uno de los problemas que genera esta situación es que los profesionales del área siguen cometiendo errores de escritura una vez graduados, incluso, en registros formales. Evidencia de ello es la afirmación de Silva (2019), quien señala que, en numerosas ocasiones, ha revisado artículos de investigación médica, en los que, reiteradamente, ha identificado una significativa cantidad de errores en su redacción y ortografía.

En el tercer lugar de los ítems con menor nivel de logro se encuentra la dimensión "Cohesión interoracional" (20,11\% de éxito promedio). Este bajo porcentaje evidencia que los estudiantes de Medicina tienen dificultadas para emplear diversos mecanismos de cohesión para 
establecer relaciones cohesivas entre las oraciones de sus textos. Por ejemplo:

[10] En ambas visitas, se observó un ambiente tranquilo y ordenado en el barrio, no se observaba basura ni residuos y se pudo observar que el servicio de recolección de basura pasaba casa por casa, además de varias veces a la semana [MED_UCN_21].

Es importante señalar que la mayoría de los problemas de uso de mecanismos de cohesión correspondían a casos de repeticiones léxicas de distintos tipos de palabras (verbos y sustantivos, principalmente). Este problema, creemos, podría deberse a la inmediatez con la que debe redactarse una Ficha Clínica, pues se escribe a medida que el paciente va relatando los hechos. La falta de diversidad léxica podría transferirse al momento de producir otros géneros profesionales como el Estudio Familiar, donde también se advierte el problema comentado. En muy pocos casos, encontramos otros tipos de problemas de mecanismos de cohesión, como, por ejemplo, reformulación o pronominalización.

Por último, el ítem "Normas sintácticas" $(31,61 \%)$ se encuentra en el cuarto lugar de las dimensiones no logradas en el área de Medicina. Este da cuenta de las dificultades para elaborar un texto libre de vicios idiomáticos. Un ejemplo de este tipo de error es:

[11] Además las actividades del Partido la mantiene entretenida y activa [MED_UCN_20].

Como se advierte en el ejemplo, se produce un error de concordancia de número. Este, de alguna manera, es sorprendente o inesperado, pues se ha identificado también en la producción de textos de niños de tercer año de Educación Primaria (Granados y Torres, 2016). Ello evidencia que los estudiantes de Medicina, aún en la Educación Terciaria, mantienen los problemas de escritura propios de primaria.

En síntesis, en Medicina, las dimensiones con mayor dificultad se agrupan bajo la macrodimensión "Normativa" y "Estructura global y oracional". Al respecto, resulta llamativo que los errores de "Normativa" sigan siendo frecuentes, incluso, en el caso de la producción de textos científicos por parte de médicos titulados (Silva, 2019). Ello sugiere 
una falta de preocupación o desconocimiento general de la norma de la lengua española, hecho que sorprende, pues se trata de personas con una alta cantidad de años de formación profesional.

Como se pudo ver en los resultados presentados, a pesar de que las rúbricas de Derecho y Medicina son instrumentos diferentes, creados sobre la base de distintos insumos disciplinares, existen ítems coincidentes. Así, si establecemos una comparación entre ambas disciplinas en el desempeño de estos ítems comunes, los resultados son los que se presentan en el Gráfico 3:

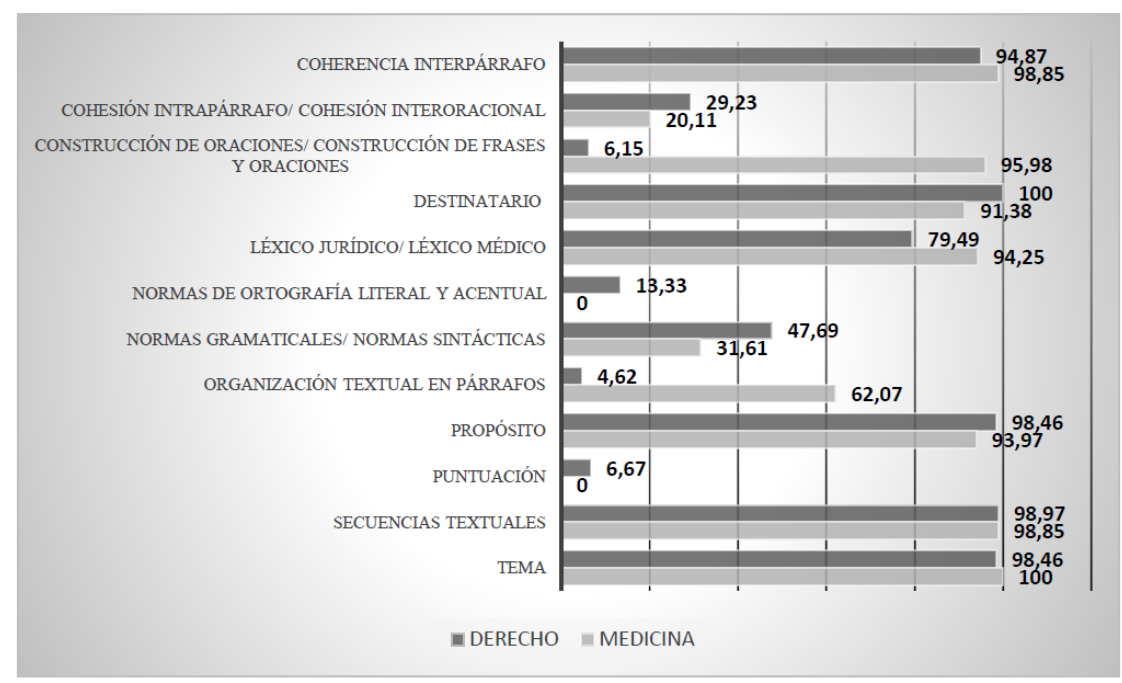

Gráfico 3 - Comparación de resultados entre disciplinas

Es importante señalar que las dimensiones que aparecen en el eje Y del Gráfico 3 con dos nombres separados por una línea oblicua son totalmente equivalentes entre Medicina y Derecho, pero reciben denominaciones diferentes para adaptar cada ítem a las distintas disciplinas (por ejemplo, "léxico jurídico/léxico médico") o a las sugerencias de los jueces expertos que participaron en el proceso de construcción de las rúbricas (por ejemplo, "normas gramaticales/ sintácticas”). En cuanto a los datos presentados, advertimos que, de las doce dimensiones que comparten ambas disciplinas, los textos de Derecho logran un mejor desempeño que en Medicina en siete de ellas, lo que equivale al $58,33 \%$. 
Más concretamente, los textos de Derecho evidencian mejor desempeño en todos los ítems que tienen que ver con normativa (ortografía, gramática y puntuación). Esto podría atribuirse a que ambas disciplinas son sumamente diferentes en muchos aspectos, entre ellos, la utilización de la escritura como un medio para cumplir con sus respectivos propósitos comunicativos. Así, Derecho es un área humanista, en la que el uso del lenguaje es fundamental, sobre todo, el dominio de las habilidades de escritura que, según Kosse y ButleRitchie (2003), es vital para la práctica de la ley. En Medicina, en cambio, la función comunicativa es más práctica, sobre todo, cuando se trata de Fichas Clínicas, que corresponden al tipo de texto en el que se comenten más errores de este tipo. A lo anterior se debe sumar que, en Medicina, como ya mencionamos, el uso de la ortografía y la puntuación es irrelevante para algunos docentes.

También, los textos de Derecho obtienen mejores resultados que Medicina en dos de los tres componentes de la situación retórica, a saber, "Destinatario" y "Propósito". Las producciones de Medicina, por su parte, evidencian un mejor nivel de logro en la dimensión "Tema". Los altos puntajes obtenidos por los estudiantes de Derecho en el ítem de "Destinatario" podrían deberse a la importancia de los distintos destinatarios de los textos que ellos escriben. Estos textos no solo son leídos por integrantes de su propia comunidad disciplinar, sino también por personas externas a ella como, por ejemplo, las partes que están involucradas en cada uno de sus casos, quienes también deben comprender la información que se presenta en los textos. Esto es evidenciado por Meza et al. (2020), quienes, al entrevistar abogados, descubren que, para muchos de ellos, uno de los elementos fundamentales a la hora de integrar la escritura clara en el ámbito jurídico está en relación con la capacidad de reconocer las características de la audiencia, identificando, específicamente, el nivel de conocimiento que esta posee en torno a cierta temática.

Con respecto al ítem de "Propósito", los altos puntajes podrían deberse a la especificidad y claridad de los propósitos comunicativos que poseen cada uno de los textos del área del Derecho que deben escribir sus estudiantes. En el caso de Medicina, el alto porcentaje en el ítem de "Tema" puede deberse a que los textos revisados (Ficha 
clínica y Estudio de Familia) abordan contenidos muy específicos que evitan que los estudiantes divaguen en otros tópicos.

En los ítems asociados con la estructura global y oracional, los textos de Derecho alcanzan mayor porcentaje de logro que Medicina en la "Cohesión intrapárrafo" y en las "Secuencias textuales". Mientras que Medicina muestra mejor desempeño en "Coherencia interpárrafo", "Construcción de frases y oraciones" y "Organización textual en párrafos". Además, en el ámbito de la comunicación del conocimiento especializado, los textos analizados de Medicina obtienen un mejor resultado que Derecho en el ítem que apunta a la utilización del léxico de la disciplina.

\section{Conclusiones}

En primer lugar, el artículo presentado cumple con el objetivo propuesto, puesto que hemos logrado identificar los problemas de escritura más frecuentes de los estudiantes de Derecho y Medicina de dos universidades regionales chilenas, al producir textos profesionales con fines académicos. Además, para mayor claridad, estos problemas fueron ejemplificados con fragmentos de textos reales.

En el caso de Derecho, todas las dimensiones no logradas corresponden a elementos asociados a la norma de escritura o la organización textual, vale decir, a aspectos puramente lingüísticos. Así, ninguna de las dimensiones más cercanas a la disciplina se encuentra entre las no logradas. Esto mismo ocurre en Medicina, disciplina en la que las dimensiones no logradas son del ámbito de la normativa y de la cohesión interoracional. Esto sugiere que los estudiantes han sido muy bien formados en los aspectos disciplinares especializados de la producción de textos jurídicos y médicos, pero no en los aspectos que dicen relación con la escritura en general.

Ahora bien, algunos de los problemas de escritura identificados en Medicina se pueden atribuir al hecho de que es un área de la ciencia en la que las habilidades lingüísticas podrían entenderse como secundarias y también a que algunos profesores entrevistados declaran que la escritura no es importante. Pese a ello, concordamos con Benavent e Iscla (2002, p. 399) cuando señalan que "la clásica distinción entre 
saberes de ciencias y saberes de letras no debe hacernos olvidar que la ciencia médica, además de que se aprende leyendo, se comunica siempre mediante la escritura". En este sentido, enseñar las formas de transmisión del conocimiento en una disciplina particular no recae únicamente en el docente experto en lengua, sino que también debiera convertirse en un trabajo conjunto entre este y el experto disciplinar (López-Gil y Molina, 2018).

En el análisis comparativo entre disciplinas, se evidencia que son muy frecuentes los problemas de ortografía y puntuación. Al respecto, es importante señalar que la función pragmática de estos elementos es distinta en ellas. Así, la sanción social de cometer una falta en una Ficha clínica y en una Demanda es diferente. En el caso de la Ficha clínica, no hay mayor sanción que reconocer que el médico ha incurrido en un error de escritura; en una Demanda, en cambio, se puede rechazar el documento por faltas ortográficas, como señala uno de nuestros entrevistados de Derecho, lo que retrasa el proceso judicial (Meza y González, 2021).

En relación con lo anterior, desde nuestro punto de vista, los aspectos lingüísticos evaluados en los textos son elementos básicos que cualquier estudiante universitario debiese dominar. Más aún si consideramos algunas variables como: el alto puntaje de ingreso de los estudiantes de Derecho y Medicina (DEMRE, 2018), el hecho de que los textos analizados se produjeron por estudiantes que cursan los últimos años de su formación de pregrado y el prestigio social que poseen ambas carreras en Chile. Además, debemos considerar que, en estas áreas, un problema de comprensión o interpretación, dado por las insuficiencias en la redacción de los textos, puede tener consecuencias sociales gravísimas. Así, en el caso de Derecho, como afirma Cazorla (2013, p. 18), "los debates jurídicos son normalmente debates sobre palabras". Por su parte, en Medicina, Bitrán et al. (2009) reafirman esta idea, mencionando que un malentendido puede tener graves consecuencias individuales y sociales.

Creemos que este estudio puede ser útil para la realización de intervenciones didácticas desde una perspectiva de alfabetización disciplinar, con base en una investigación empírica y situada, vale decir, con datos reales de disciplinas específicas. Esto, dado que, como sugieren 
Parodi et al. (2010: 49), para ello "es requisito fundamental contar con una descripción de los textos que se leen y que circulan en los ámbitos de especialidad". De esta forma, la detección de los problemas en la escritura de estudiantes de Derecho y Medicina que aquí presentamos se convierte en un insumo para la elaboración, no solo de material didáctico que emerge de las reales necesidades de los estudiantes, sino también para la creación de cursos y programas de escritura. Así, a partir de la comprensión de las características específicas del discurso de la Medicina y el Derecho, se podrían desarrollar propuestas didácticas concretas para los docentes expertos en lengua y de especialidad. Ello contribuirá a alfabetizar a los estudiantes en lengua escrita especializada para que superen exitosamente su formación académica y, además, logren un desempeño idóneo en el mundo laboral.

En cuanto a las proyecciones, creemos importante abrir esta investigación a otras áreas, como, por ejemplo, las carreras de pedagogía, pues son los profesores quienes forman a los estudiantes en la producción de textos. Además, la rúbrica utilizada es un instrumento flexible y replicable que se podría adaptar a otros textos y otras disciplinas.

\section{Referencias}

AHMAD, Zulfiqar. 2019. Differences between Students' Linguistic Knowledge and Text Production Ability: A Case of the Use of Cohesion as a Resource of Texture in Academic Writing. World Journal of English Language, 9/2: 55-63.

ALCARAZ, Enrique et al. 2014. El español jurídico. Barcelona: Ariel.

ARAUJO, Zandra. 2018. Caracterización de las respuestas a las consignas de los exámenes reprobados. Ingresantes-Escuela de Derecho (A2016). Educere, 22/72: 307-315.

ARECHABALA, María Cecilia et al. 2011. Géneros discursivos y errores más frecuentes en los informes académicos de estudiantes de enfermería. Invest Educ Enferm, 29/3: 400-406.

BADLEY, Graham. 2009. Academic writing as shaping and reshaping. Teaching in Higher Education, 14/2: 209-219. doi: 10.1080/13562510902757294. 
BASSI, Javier. 2016. La escritura académica: 30 errores habituales y cómo abordarlos. Quaderns de Psicología, 18/1: 119-142.

BENAVENT, Rafael; ISCLA, Alberto. 2002. Vicios del lenguaje médico y defectos de estilo en la escritura cientificomédica (I). Piel, 17/9: 399-404. doi:10.1016/s0213-9251(02)72628-2.

BITRÁN, Marcela. et al. 2009. Mejoría en las habilidades de comunicación escrita de estudiantes de medicina: Impacto de un taller de escritura. Revista médica de Chile, 137/5: 617-624. https://dx.doi. org/10.4067/S0034-98872009000500004

BUSTOS, José. 2011. Errores discursivos y estilísticos en la expresión escrita: categorización y valoración. Quaderns de Filología. Estudis Lingüistics, 16: 41-64.

CARLINO, Paula. 2004. El proceso de escritura académica: Cuatro dificultades de la enseñanza universitaria. Educere, 8/26: 321-327. . 2005. Escribir, leer, y aprender en la universidad. Una introducción a la alfabetización académica. Buenos Aires: Fondo de Cultura Económica.

CAZORLA, Luis. 2013. El lenguaje jurídico actual. Navarra: Thomson Reuters Aranzadi.

COFRÉ, Evelyn. 2019. Competencia y desempeño en ortografía de estudiantes de Educación Básica y de estudiantes en formación inicial docente. Tesis para optar al grado de Licenciado en Educación y al título de Profesora de Educación General Básica, mención Lenguaje y Cs. Sociales, Universidad de Concepción, Chile.

DEPARTAMENTO DE EVALUACIÓN, MEDICIÓN Y REGISTRO. 2018. Proceso de admisión 2019- Oferta Definitiva de carreras, Vacantes y Ponderaciones. Santiago de Chile: DEMRE.

ERRÁZURIZ, María Constanza. 2014. Análisis del uso de los marcadores discursivos en argumentaciones escritas por estudiantes universitarios. Perfiles educativos, 34/136: 98-117.

GAIBANI, Ahmed. 2015. An Investigation into Undergraduates' Errors in the use of the Indefinite Article at Omar Al-Mukhtar University. Advance in Language and Literary Studies, 6/6: 195-200.

GIDI, Antonio; WEIHOFEN, Henry. 2018. Legal Writing Style. Saint Paul, Minnesota: West Academic Publishing.

GRANADOS, Dora; TORRES, Patricia. 2016. Errores de escritura en español en niños de tercer grado de educación primaria. Pensamiento Psicológico, 14/2: 113-124. https://dx.doi.org/10.11144/Javerianacali. PPSI14-2.eeen 
GUNNARSSON, Britt-Louise. 2004. The multilayered structured of enterprise discourse. Information Design Journal + Document Design, 12/1: 36-48.

HALL, Beatriz; LÓPEZ, María Isabel. 2011. Discurso académico: manuales universitarios y prácticas pedagógicas. Literatura y Lingüística, 23: 167-192. doi: 10.4067/S0716-58112011000100010 HERNÁNDEZ, Roberto et al. 2006. Metodología de la investigación. México D.F.: McGraw-Hill.

IBÁÑEZ, Romualdo. 2008. El texto disciplinar y el acceso al conocimiento desde el análisis del género: ¿Regulación del conocimiento o persuasión? En: PARODI, Giovanni (Ed.). Géneros académicos y géneros profesionales: accesos discursivos para saber y hacer. Valparaíso: Ediciones Universitarias de Valparaíso.

KANOKSILAPATHAM, Budsaba. 2007. Rhetorical moves in biochemistry research articles. In: BIBER, Douglas; CONNOR, Ulla; UPTON, Thomas (Eds.). Discourse on the move. Ámsterdam: Benjamins.

KOSSE, Susan; BUTLERITCHIE, David. 2003. How Judges, Practitioners, and Legal Writing Teachers Assess the Writing Skills of New Law Graduates: A Comparative Study. Journal of Legal Education. 53: 80-102.

LÓPEZ-GIL, Karen; MOLINA, Violeta. 2018. Incidencia del trabajo colaborativo docente en la enseñanza y el aprendizaje de la escritura académica. Revista Electrónica de Investigación Educativa, 20/1: 1-13.doi: 10.24320/redie.2018.20.1.1477.

LOWE, Charles; ZEMLIANSKY, Pavel. 2010. Writing Spaces: Readings on Writings. South Carolina: Parloss Press LLC, Anderson.

MARTÍNEZ, Juan. 2015. El género Tesis Doctoral de Historia y Física: descripción y variación retórico-funcional. En Leer y escribir en contextos académicos y profesionales: géneros, corpus y métodos. En: PARODI, Giovanni; BURDILES, Gina (Eds.). Leer y escribir en contextos académicos y profesionales. Géneros, corpus y métodos. Santiago: Ariel.

MEZA, Paulina; DA CUNHA, Iria. 2019. Comunicación del conocimiento propio y relaciones discursivas en el género tesis. Sintagma. Revista de Lingüística, 31: 103-130.

MEZA, Paulina; GONZÁLEZ, Felipe. 2021. Un instrumento para evaluar la calidad lingüístico-discursiva de textos disciplinares producidos por estudiantes de Derecho. Onomázein, 51. doi: 10.7764/ onomazein.51.08. 
MEZA, Paulina; GONZÁLEZ-CATALÁN, Felipe. 2020. Propuesta de un instrumento para evaluar la calidad lingüístico-discursiva de textos disciplinares de Medicina. Logos: Revista de Lingüística, Filosofía y Literatura, 30/1: 3-17. doi: 10.15443/rl3001.

MEZA, Paulina et al. 2020. Plain writing in the legal field: An approach from the discourse of specialists. Discourse Studies. doi: $10.1177 / 1461445620906027$.

MONTOLÍO, Estrella. 2000. Manual de escritura académica. Barcelona: Ariel.

MELGUIZO, Elisabeth. 2016. Los errores ortográficos en trabajos académicos de alumnos universitarios. En: DÍEZ, Antonio; BROTONS, Vicent; ESCANDELL, Dari; ROVIRA, José (Ed.). Aprendizajes plurilingües y literarios. Nuevos enfoques didácticos. Alacant: Publicacions de la Universitat d'Alacant.

MUÑOZ, Santiago. 2017. Libro de estilo de la Justicia. Barcelona: Espasa.

OLAVE-ARIAS, Giohanny et al. (2013). Deserción universitaria y alfabetización académica. Educ. Educ., 16/3: 455-471.

PARODI, Giovanni. 2007. El discurso especializado escrito en el ámbito universitario y profesional: Constitución de un corpus de estudio. Revista Signos, 40/63: 147-178.

. 2009. El Corpus Académico y Profesional del Español PUCV2006: semejanzas y diferencias entre los géneros académicos y profesionales. Estudios Filológicos. 44: 123-147.

PARODI, Giovanni et al. 2010. Alfabetización académica y profesional en el siglo XXI: Leer y escribir desde las disciplinas. Santiago: Ariel.

PÉREZ, Mercedes et al. 2012. Estrategia para desarrollar habilidades ortográficas en los estudiantes de Tecnología de la Salud. Humanidades Médicas, 12/2: 337-346.

RICO, Ana María; NÍKLEVA, Dimitrinka. 2016. Análisis de la competencia lingüístico-discursiva escrita de los alumnos de nuevo ingreso del Grado de Maestro en Educación Primaria. Revista Signos, 49/90: 48-70. doi: 10.4067/S0718-0934201600010000

RODRÍGUEZ, Blanca; GARCÍA, Laura. 2015. Escritura de textos académicos: dificultades experimentadas por escritores noveles y sugerencias de apoyo. Revista de Investigación Educativa, 20: 249-265.

SABAJ, Omar. 2009. Descubriendo algunos problemas en la redacción de Artículos de Investigación Científica (AIC) de alumnos de postgrado. Revista Signos. Estudios de Lingüística, 42/69: 107-127. doi: 10.4067/ S0718-09342009000100006

SÁNCHEZ, Carlos. 2005. Los problemas de redacción de los estudiantes costarricenses: una propuesta de revisión desde la lingüística del texto. Filología y Lingüística, 31/1: 267-295. 
SILVA, Carlos. 2019. El buen uso del idioma español en la literatura médica. Revista médica de Chile, 147/5: 643-649. doi: 10.4067/ S0034-98872019000500643.

SOTOMAYOR, Carmen et al. 2013. Caracterización de problemas ortográficos recurrentes en alumnos de escuelas municipales chilenas de $3^{\circ}, 5^{\circ}$ y $7^{\circ}$ básico. Revista Signos, 46/81: 105-131.

STAPLES, Shelley et al. 2016. Academic Writing Development at the University Level: Phrasal and Clausal Complexity Across Level of Study, Discipline, and Genre. Writing Communication, 33/2: 149-183. doi: $10.1177 / 0741088316631527$

TAPIA, Mónica et al. 2003. Aplicación de una pauta diseñada para evaluar informes académicos universitarios. Revista Signos, 36/54: 249-257. doi: 10.4067/S0718-09342003005400009.

URIBE, Olga; CARRILLO, Stefany. 2014. Relación entre la lectoescritura, el desempeño académico y la deserción estudiantil. Entramado, 10/2: 272-285.

VALENCIA, Claudia. 2014. Errores de ortografia acentual y literal frecuentes en textos informativos producidos por estudiantes de educación superior técnico profesional en Santiago de Chile. Tesis para optar al grado de Magíster en Lingüística, mención Lengua Española, Universidad de Chile, Chile.

Recebido em: 25/02/2020

Aprovado em: 31/12/2020 